\title{
Strong Electron-Phonon and Band Structure Effects in the Optical Properties of High Pressure Metallic Hydrogen
}

\author{
Miguel Borinaga, ${ }^{1,2}$ Julen Ibañez-Azpiroz, ${ }^{3}$ Aitor Bergara, ${ }^{1,2,4}$ and Ion Errea ${ }^{2,5}$ \\ ${ }^{1}$ Centro de Física de Materiales CFM, CSIC-UPV/EHU, Manuel Lardizabal Pasealekua 5 , \\ 20018 Donostia/San Sebastián, Basque Country, Spain \\ ${ }^{2}$ Donostia International Physics Center (DIPC), Manuel Lardizabal Pasealekua 4, \\ 20018 Donostia/San Sebastián, Basque Country, Spain \\ ${ }^{3}$ Peter Grünberg Institute and Institute for Advanced Simulation, Forschungszentrum Jülich \& JARA, D-52425 Jülich, Germany \\ ${ }^{4}$ Departamento de Física de la Materia Condensada, University of the Basque Country (UPV/EHU), \\ 48080 Bilbao, Basque Country, Spain \\ ${ }^{5}$ Fisika Aplikatua 1 Saila, Bilboko Ingeniaritza Eskola, University of the Basque Country (UPV/EHU), \\ Rafael Moreno "Pitxitxi” Pasealekua 3, 48013 Bilbao, Basque Country, Spain
}

(Received 4 July 2017; published 30 January 2018)

\begin{abstract}
The recent claim of having produced metallic hydrogen in the laboratory relies on measurements of optical spectra. Here, we present first-principles calculations of the reflectivity of hydrogen between 400 and $600 \mathrm{GPa}$ in the $I 4_{1} /$ amd crystal structure, the one predicted at these pressures, based on both timedependent density functional and Eliashberg theories, thus, covering the optical properties from the infrared to the ultraviolet regimes. Our results show that atomic hydrogen displays an interband plasmon at around $6 \mathrm{eV}$ that abruptly suppresses the reflectivity, while the large superconducting gap energy yields a sharp decrease of the reflectivity in the infrared region approximately at $120 \mathrm{meV}$. The experimentally estimated electronic scattering rates in the $0.7-3 \mathrm{eV}$ range are in agreement with our theoretical estimations, which show that the huge electron-phonon interaction of the system dominates the electronic scattering in this energy range. The remarkable features in the optical spectra predicted here encourage extending the optical measurements to the infrared and ultraviolet regions as our results suggest optical measurements can potentially identify high-pressure phases of hydrogen.
\end{abstract}

DOI: 10.1103/PhysRevLett.120.057402

In 1935, Wigner and Huntington predicted that, at high pressure, hydrogen molecules would dissociate yielding a metallic compound similar to the alkalis [1]. Metallic hydrogen is expected to be a wonder material as it may superconduct at ambient temperature [2-8]. Despite a huge experimental effort in the last years that has characterized the phase diagram of hydrogen up to very high pressures [9-20], metallic hydrogen has remained elusive. However, the long standing quest might have come to an end as, early 2017, Dias and Silvera reported the first ever laboratoryproduced sample of metallic hydrogen [21].

Metallic hydrogen was claimed to have been observed as the sample became reflective above $495 \mathrm{GPa}$ [21]. The claim remains controversial as doubts on the pressure calibration have been raised and a semiconducting sample may also be reflective [22-25]. Moreover, raw reflectance data in Ref. [21] show a sharp decrease for photon energies larger than $2 \mathrm{eV}$ whose origin is not totally understood even though it was first attributed to absorption of diamond [26]. Anyway, the claim of having produced metallic hydrogen comes after previous works in which the first signals of its existence were present or close to appearing [20,27]. Thus, reproducibility of the experiment and exhaustive characterization of the system are clearly the next challenge. Characterizing hydrogen under pressure is extremely difficult due to the limitations imposed on conventional techniques. Unavailability of neutron scattering and $\mathrm{x}$-ray diffraction experiments for extremely highpressure hydrogen samples in diamond anvil cells make the use of alternative techniques imperative. Many of the already known solid hydrogen phases have been characterized by comparing Raman scattering and infrared (IR) absorption data to theoretical calculations [9,14-17,20,28]. Comparing optical reflectance spectra to theoretical estimations is, indeed, another option [21,29].

In this Letter, we report an exhaustive characterization of the optical properties from the IR to the extreme ultraviolet (UV) of metallic hydrogen between 400 and $600 \mathrm{GPa}$ in the atomic $I 4_{1} /$ amd phase, the structure predicted for hydrogen at these pressures [30,31]. Our fully first-principles analysis based on density-functional theory (DFT) sheds light into the regime measured by Dias and Silvera [21], from 0.75 to $3 \mathrm{eV}$ photon energies, showing that, in this range, the electronic relaxation time is dominated by the huge electron-phonon interaction. Besides, our calculations predict a complex reflectance spectrum not expected a priori for a free-electron-like alkali metal. Our calculations reveal a sharp onset of the optical conductivity in the 
IR region induced by the very large superconducting gap of atomic hydrogen [7]. This suggests that reflectivity measurements at temperatures below the superconducting critical temperature $T_{c}$, predicted to be $300 \mathrm{~K}$ [7], might be used to measure optically $T_{c}$ and the superconducting gap as it occurs, for instance, in cuprates [32], alkali-metaldoped fullerenes [33], and the recently discovered [34] record superconductor $\mathrm{H}_{3} \mathrm{~S}$ [35]. On the other extreme, the UV regime exhibits a pronounced loss of reflectance due to the presence of a non-free-electron-like interband plasmon. For comparison, we have also studied the optical spectra of the molecular metallic $\mathrm{Cmca}-4$ phase, which has recently been suggested as the structure that describes a new phase that emerges around $300 \mathrm{GPa}$ and $370 \mathrm{~K}$ [36]. The different features on the reflectivity indicate that the optical spectra can be potentially used to identify high-pressure phases of hydrogen. Therefore, our results provide clear means of characterizing metallic hydrogen via these singular features, strongly encouraging the extension of experimental optical measurements [21] to broader regimes.

The central quantity addressed in this work is the frequency dependent reflectivity, which, for normal incident light in a medium with refractive index $n$, can be written as

$$
R(\omega)=\left|\frac{\sqrt{\varepsilon(\omega)}-n}{\sqrt{\varepsilon(\omega)}+n}\right|^{2} .
$$

As we are dealing with a metal, we have calculated the relative dielectric function $\varepsilon(\omega)$ by combining timedependent DFT [37-39] (TDDFT), which realistically incorporates the actual electronic structure into the dielectric function, and isotropic Migdal-Eliashberg (ME) equations $[40,41]$, which take into account how an excited electron can decay due to the electron-phonon interaction (see the Supplemental Material [42] for a more detailed description of the methods and the calculation procedure). ME equations are solved with different $\tau_{\text {imp }}^{-1}$ impurity scattering rates. This enables us to properly account for the optical features of metallic and presumably superconducting hydrogen not only in the visible and UV regions, but also in the IR regime, which could be strongly affected due to the presence of the superconducting gap $\Delta_{0}[32,33,35]$. All the calculations presented in this Letter are performed at $500 \mathrm{GPa}$, where metallic hydrogen is predicted to adopt the $I 4_{1} /$ amd crystal structure [30,31]. Calculations performed at 400 and 600 GPa presented in the Supplemental Material [42] show a very weak pressure dependence of the reflectivity as only minor quantitative, but not qualitative, differences are observed. Thus, the analysis presented here holds at higher and lower pressures.

In Fig. 1(a), we show the calculated reflectivity of $I 4_{1} /$ amd hydrogen in vacuum $[n=1$ in Eq. (1)] in the low temperature limit $(50 \mathrm{~K})$ at $500 \mathrm{GPa}$, for both the normal and superconducting states. We find two different regimes for the optical spectra: the IR regime $(\omega<1 \mathrm{eV})$, where the effects related to scattering with phonons and impurities dominate, and the visible and UV regime $(\omega>1 \mathrm{eV})$, where electronic band structure effects start to play a role.

The inset in Fig. 1(a) shows the low temperature limit of the reflectivity at $500 \mathrm{GPa}$ for IR radiation. Clean hydrogen $\left(\tau_{\text {imp }}^{-1}=0\right)$ in the normal state (which can be obtained by setting $\Delta_{0}=0$ in ME equations) reflects all the incoming light until phonons start contributing substantially to $\alpha^{2} F(\omega)$ above $\sim 100 \mathrm{meV}$. When impurity scattering is taken into account, reflectivity decreases from one right from the beginning, reaching a small plateau $(\sim 0.99$ for $\tau_{\text {imp }}^{-1}=200 \mathrm{meV}$ ) until scattering with phonons starts to be relevant. In the superconducting state, the reflectivity is equal to unity below $2 \Delta_{0}=122 \mathrm{meV}$ even when impurity scattering is taken into account, as that is the amount of energy required to break a Cooper pair and make electrons contribute to the optical conductivity. This can be clearly seen in Fig. 2(b), where $\operatorname{Im} \varepsilon$ is strictly zero below $2 \Delta_{0}$ (except the zero frequency contribution coming from the dc conductivity of the Cooper pairs) abruptly increasing at

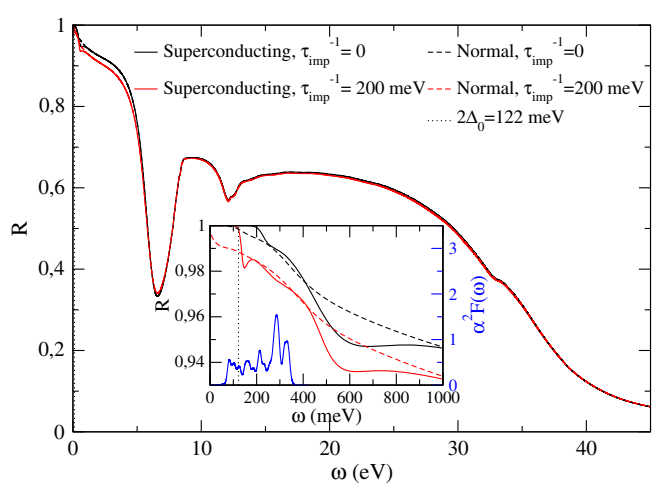

(a)

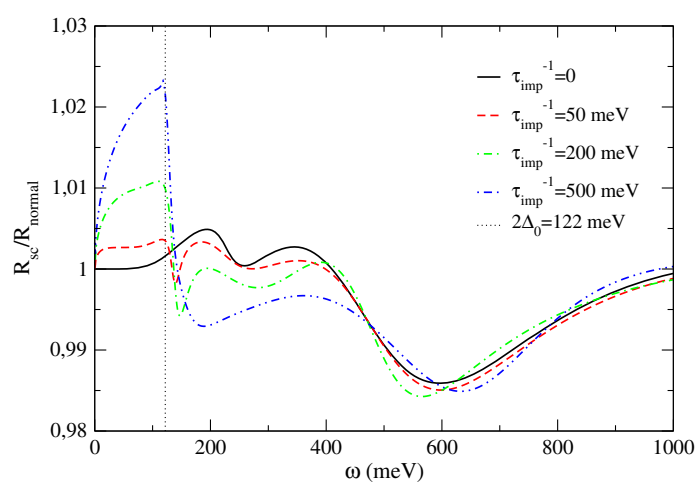

(b)

FIG. 1. (a) Reflectivity of $I 4_{1} /$ amd hydrogen in vacuum at $50 \mathrm{~K}$ and $500 \mathrm{GPa}$ for different impurity scattering rates both in the normal and superconducting states. The inset shows the same curves at the low energy regime along with the electron-phonon spectral function $\alpha^{2} F(\omega)$. (b) Ratio between superconducting and normal state reflectance of $I 4_{1} /$ amd hydrogen in vacuum at $50 \mathrm{~K}$ and $500 \mathrm{GPa}$ for different impurity scattering rates. 


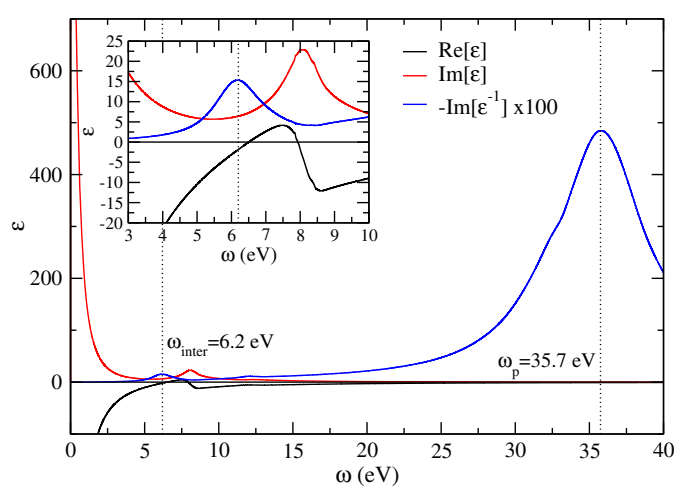

(a)

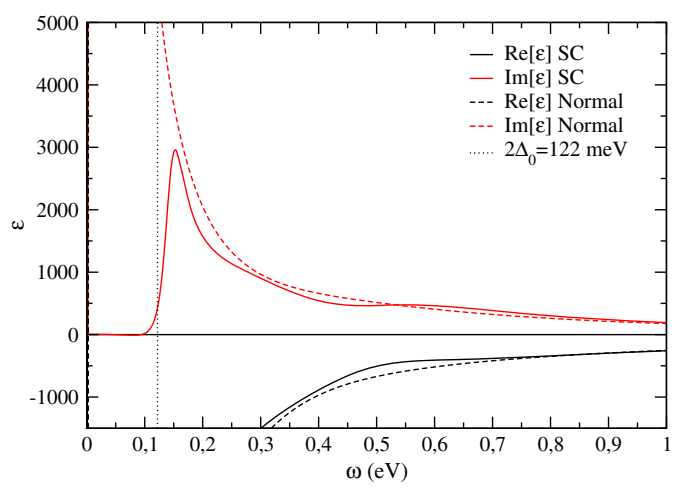

(b)

FIG. 2. (a) $\operatorname{Re} \varepsilon, \operatorname{Im} \varepsilon$ and $-\operatorname{Im} \varepsilon^{-1}$ for $I 4_{1} /$ amd hydrogen at $50 \mathrm{~K}$ and $500 \mathrm{GPa}$ for $\tau_{\text {imp }}^{-1}=200 \mathrm{meV}$ in the normal state. The inset shows the same curves zoomed in the interband plasmon region. (b) Real and imaginary parts of the dielectric function of $I 4_{1} / a^{2} d$ hydrogen at $50 \mathrm{~K}$ and $500 \mathrm{GPa}$ in the IR region for $\tau_{\mathrm{imp}}^{-1}=200 \mathrm{meV}$ in both the normal and superconducting (SC) states.

larger energies. While for $\tau_{\mathrm{imp}}^{-1}=200 \mathrm{meV}$ the gap is clearly observable due to the sudden decrease of $R$, it is not the same for the clean case; in order to have electrons contributing to the optical conductivity, one needs both to break Cooper pairs and scattering with phonons to conserve both energy and momentum. The necessity of impurities for observing the superconducting gap optically is already well known and becomes more evident if one plots the ratio between superconducting and normal state reflectance $\left(R_{s c} / R_{n}\right)$ for different $\tau_{\text {imp }}^{-1}$ values [Fig. 1(b)]. This figure clearly shows the emergence of a sharp decrease at $\omega=$ $2 \Delta_{0}$ only when impurity scattering is included. The gap is observable even in the clean limit $\left(\tau_{\text {imp }}^{-1}=50 \mathrm{meV}<2 \Delta_{0}\right)$, but the drop in $R_{s c} / R_{n}$ is more notorious as one approaches the dirty limit $\left(\tau_{\text {imp }}^{-1} \gg 2 \Delta_{0}\right)$.

The reflectivity above $\omega=1 \mathrm{eV}$ for normal and superconducting states is almost identical [see Fig. 1(a)]. The effect of impurity scattering up to $5 \mathrm{eV}$ only yields quantitative differences keeping the shape of the reflectivity curve unaltered. Actually, for $\omega>5 \mathrm{eV}$, all the curves converge into one, suggesting electronic scattering is dominated by electronic band structure effects rather than phonons and impurities. Remarkably, in this UV regime, the reflectivity sharply decreases from a high $\sim 0.95$ value in the visible range $(\omega=[1.6-3.3] \mathrm{eV})$ to $\sim 0.3$ at $\omega=6.5 \mathrm{eV}$. This stark reduction of the reflectance is a consequence of light absorption due to the presence of an interband plasmon not expected a priori for a simple free-electron-like alkali metal. This is demonstrated in Fig. 2(a), where we display the calculated dielectric function. The interband plasmon emerges around the energy where $\operatorname{Re} \varepsilon$ vanishes and $\operatorname{Im} \varepsilon$ remains low. This induces a clear peak in $-\operatorname{Im} \varepsilon^{-1}$ at $\omega_{\text {inter }}=$ $6.2 \mathrm{eV}$ as shown in Fig. 2(a), which coincides with the drastic drop in the reflectivity. We label this plasmon as interband because it is a consequence of the interband transitions of around $8.2 \mathrm{eV}$ that occur close to the $N$ point (see band structure in Fig. 3). Consequently, $\operatorname{Im} \varepsilon$ shows a clear peak at
$8.2 \mathrm{eV}$, which, due to Kramers-Kronig relations, makes the real part pass through 0 at $6.5 \mathrm{eV}$ and create the interband plasmon. Even if the band structure of $I 4_{1} /$ amd is not far from the free-electron limit, the large gap opened by the electron-ion interaction at the $N$ point [7] suffices to induce the presence of an interband plasmon not expected for a freeelectron-like metal. Thus, metallic hydrogen in the $I 4_{1} /$ amd phase is another example in which the departure from the free-electron-like character makes interband plasmons emerge and abruptly modify the optical properties, as it occurs in other simple compounds under pressure, such as $\mathrm{Li}$ [53-55], $\mathrm{Ca}$ [43], $\mathrm{Na}$ [44,56], $\mathrm{Rb}, \mathrm{Cs}$ [57], and $\mathrm{AlH}_{3}$ [58].

Apart from the interband plasmon, we find that metallic hydrogen shows the expected free-electron plasmon at $\omega_{p}=35.7 \mathrm{eV}$, which is responsible for the final decrease of the reflectivity at the extreme UV regime. This value is in good agreement with both the Drude-model estimate of $\omega_{p}=\sqrt{4 \pi N}=35.0 \mathrm{eV}$, where $N$ is the total electronic density, and the $33.2 \pm 3.5 \mathrm{eV}$ experimental value obtained by Dias and Silvera [26]. This experimental fit also provided a

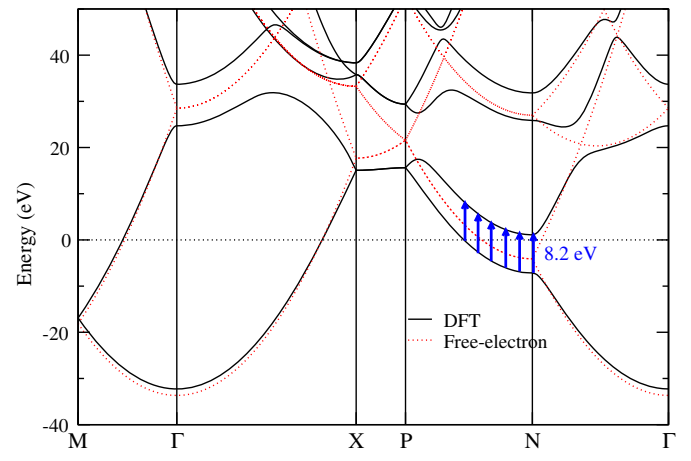

FIG. 3. Electronic band structure of $I 4_{1} /$ amd hydrogen at $500 \mathrm{GPa}$. Interband transitions around the $N$ point that yield a peak in $\operatorname{Im} \varepsilon$ are marked with blue arrows. The DFT bands are compared to the free-electron band structure. The Fermi level is at $0 \mathrm{eV}$. 
$\tau^{-1}=1.1 \pm 0.2 \mathrm{eV}$ estimate for the electronic scattering rate [26]. In order to shed some light into these experimental values, we fit our calculated dielectric function to a Drude model with frequency-dependent $\omega_{p}(\omega)$ and $\tau^{-1}(\omega)$, which are displayed in Fig. 4(a) (see Supplemental Material [42] for details). In the $\omega=[0.7-3] \mathrm{eV}$ range, our results yield $\omega_{p} \sim 21 \mathrm{eV}$ and $\tau^{-1} \sim 0.6-1 \mathrm{eV}$ at $5 \mathrm{~K}$ for a clean sample, with impurities shifting $\tau^{-1}$ upwards. Our estimated $\tau^{-1}$ in the measured frequency range is in good agreement with the experiment and clearly shows that its large value is mainly due to the strong electron-phonon interaction, which highlights the fact that we are dealing with a superconductor with a very large $T_{c}$. Indeed, the electron-electron scattering contribution to $\tau^{-1}$ is negligible in this frequency range as shown by the pure TDDFT calculation. The value obtained for $\omega_{p}$ is considerably lower than the one obtained by Silvera and Dias [21,26], but it is consistent with the $\omega_{p}^{\text {intra }}=$ $\sqrt{4 \pi N_{\text {intra }}}=22.6 \mathrm{eV}$ value, where $N_{\text {intra }}$ is the electronic density contributing to intraband transitions (see Supplemental Material [42] for details). Nonetheless, in this low energy $\left(\omega \ll \omega_{p}\right)$ regime, very different $\omega_{p}$ values still provide a good fitting to the experimental data, while $\tau^{-1}$ remains almost unaltered (see Supplemental Material [42]). Thus, we consider the $\tau^{-1}$ value obtained experimentally $[21,26]$ to be more meaningful than the plasma frequency, because it is, indeed, this parameter that determines how much the reflectivity deviates from one for $\omega \ll \omega_{p}$.

In Fig. 4(b), we show how the raw experimental values in Ref. [21] compared to our calculations at 5 and $83 \mathrm{~K}$, with reflectivity calculated for a hydrogen-diamond interface by using the refractive index of $n=2.41$ of diamond instead of $n=1$ in Eq. (1). Our results compare well at $5 \mathrm{~K}$ to the two lowest frequency experimental data points, which are the ones considered for fitting $\omega_{p}$ and $\tau^{-1}$ with the Drude model as the other points might have been affected by absorption of light by diamond $[21,26]$. The sharp offset of reflectivity due to the superconducting gap lays off the IR absorption range of diamond, and should be measurable in consequence. UV absorption of diamond, however, would eclipse the minimum of the reflectivity predicted here at $6.5 \mathrm{eV}$ due to the presence of the interband plasmon, since above the indirect electronic band gap of $5.47 \mathrm{eV}$ (at zero pressure) diamond is no longer transparent. Nevertheless, the sharp decrease associated to such a plasmon starts before the absorption onset and should be observable using pure diamond. However, impurities in diamond could be responsible for light absorption at lower energies, even in the visible region [26]. In order to disentangle whether the reflectivity drop observed experimentally is a consequence of diamond absorption or reflects the presence of the intraband plasmon we are predicting here, a proper characterization of the optical properties of diamond at the experimental conditions is required.

In the experimental region, our calculated reflectivity and scattering rate values are practically temperature independent (see Fig. 4). Our calculations predict that temperature only affects the region within some meVs around the superconducting gap. This indicates that the temperature dependence shown in the experiments cannot be explained with the increase of phonon occupation in the system.

Motivated by uncertainties in the reported pressure of the experiment [22-25], we have extended our analysis in two directions. First, we have calculated the optical spectra for $I 4_{1} /$ amd hydrogen at 400 and $600 \mathrm{GPa}$ and only found minor quantitative differences with respect to the $500 \mathrm{GPa}$ results analyzed in detail here, so that the analysis holds. The energy of the interband plasmon at $400 \mathrm{GPa}$ is $5.5 \mathrm{eV}$ and $6.6 \mathrm{eV}$ at $600 \mathrm{GPa}$ (more details in the Supplemental

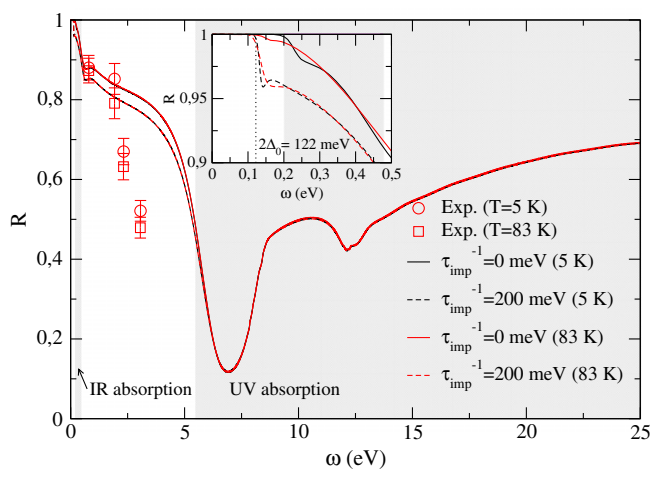

(b)

FIG. 4. (a) Frequency-dependent electronic scattering rate of $I 4_{1} /$ amd hydrogen at $500 \mathrm{GPa}$ for different impurity scattering rates and temperatures in the region in which the experiments in Ref. [21] were performed. The same curve is also obtained for the case in which the ME formalism is not considered (curve labeled as TDDFT only). The experimental $\tau^{-1}=1.1 \pm 0.2 \mathrm{eV}$ estimate is also included [26]. The inset shows the obtained frequency dependent $\omega_{p}(\omega)$ in the same energy range, together with the $\omega_{p}^{\text {intra }}$ estimate. (b) Reflectivity of a $I 4_{1} /$ amd hydrogen-diamond interface at 5 and $83 \mathrm{~K}$ at $500 \mathrm{GPa}$ for different impurity scattering rates. Diamond IR (0.2-0.47 eV) and UV (5.47 eV electronic band gap) absorption regions are shown in shaded gray. Experimental raw values [21] are included. 
Material [42]). Additionally, we have also calculated the optical spectrum of $\mathrm{Cmca}-4$ hydrogen, which has been suggested as a potential candidate of metallic molecular hydrogen [36] (see Supplemental Material [42]). According to our results, the calculated optical spectrum for both structures shows very different features, which supports the potential validity of the reflectivity to identify the structure and encourage extending optical measurements on a wider energy range. The calculated reflectivity for $\mathrm{Cmca}-4$ significantly deviates from the measured one due to the presence of enhanced interband electronic transitions in this molecular structure.

In conclusion, we have made an exhaustive analysis of the optical response properties of $I 4_{1} /$ amd metallic hydrogen from the infrared region to the extreme ultraviolet. Our results show that, in the measured energy range [21], the electronic scattering is dominated by the huge electronphonon interaction of the system. Besides, our calculations reveal a sharp onset of the optical conductivity in the infrared region induced by the very large superconducting gap and a pronounced loss of reflectance in the ultraviolet regime due to the presence of a non-free-electron-like plasmon similar to others predicted and found in alkalis and hydrides at high pressure [44,53-58]. Even though the calculated reflectivity agrees with the experiment at the two lowest energies, where the reflectivity considerably lowers due to the high electronphonon coupling, it slightly deviates at higher energies. Finally, our calculations suggest that different phases of hydrogen can potentially be distinguished from their optical spectra. Thus, our work deeply encourages further experimental research in order to extend optical measurements to both the ultraviolet and the infrared regimes. Confirming the predicted interband plasmon and measuring the superconducting gap optically would be not only of tremendous interest by itself, but also a big step towards characterizing this fascinating material.

The authors acknowledge financial support from the Spanish Ministry of Economy and Competitiveness (Grant No. FIS2016-76617-P) and the Department of Education, Universities and Research of the Basque Government and the University of the Basque Country (Grant No. IT75613). M. B. is also thankful to the Department of Education, Language Policy and Culture of the Basque Government for support (Grant No. PRE-2016-2-0134). Computer facilities were provided by the Donostia International Physics Center (DIPC). J. I. A. acknowledges the Impuls und Vernetzungsfonds der Helmholtz-Gemeinschaft Postdoc Programme.

[1] E. Wigner and H. B. Huntington, J. Chem. Phys. 3, 764 (1935).

[2] N. W. Ashcroft, Phys. Rev. Lett. 21, 1748 (1968).
[3] P. Cudazzo, G. Profeta, A. Sanna, A. Floris, A. Continenza, S. Massidda, and E. K. U. Gross, Phys. Rev. Lett. 100, 257001 (2008).

[4] J. M. McMahon and D. M. Ceperley, Phys. Rev. B 84, 144515 (2011).

[5] Y. Yan, J. Gong, and Y. Liu, Phys. Lett. A 375, 1264 (2011).

[6] E. Maksimov and D. Savrasov, Solid State Commun. 119, 569 (2001).

[7] M. Borinaga, I. Errea, M. Calandra, F. Mauri, and A. Bergara, Phys. Rev. B 93, 174308 (2016).

[8] M. Borinaga, P. Riego, A. Leonardo, M. Calandra, F. Mauri, A. Bergara, and I. Errea, J. Phys. Condens. Matter 28, 494001 (2016).

[9] M. I. Eremets and I. A. Troyan, Nat. Mater. 10, 927 (2011).

[10] P. Loubeyre, R. LeToullec, D. Hausermann, M. Hanfland, R. J. Hemley, H. K. Mao, and L. W. Finger, Nature (London) 383, 702 (1996).

[11] S. Deemyad and I. F. Silvera, Phys. Rev. Lett. 100, 155701 (2008).

[12] A. B. Stanimir, E. Schwegler, T. Ogitsu, and G. Galli, Nature (London) 431, 669 (2004).

[13] H.-k. Mao and R. J. Hemley, Rev. Mod. Phys. 66, 671 (1994).

[14] C.-S. Zha, Z. Liu, and R. J. Hemley, Phys. Rev. Lett. 108, 146402 (2012).

[15] A. F. Goncharov, E. Gregoryanz, R. J. Hemley, and H.-k. Mao, Proc. Natl. Acad. Sci. U.S.A. 98, 14234 (2001).

[16] R. T. Howie, C. L. Guillaume, T. Scheler, A. F. Goncharov, and E. Gregoryanz, Phys. Rev. Lett. 108, 125501 (2012).

[17] C.-s. Zha, Z. Liu, M. Ahart, R. Boehler, and R. J. Hemley, Phys. Rev. Lett. 110, 217402 (2013).

[18] R. T. Howie, P. Dalladay-Simpson, and E. Gregoryanz, Nat. Mater. 14, 495 (2015).

[19] P. Loubeyre, F. Occelli, and R. LeToullec, Nature (London) 416, 613 (2002).

[20] P. Dalladay-Simpson, R. T. Howie, and E. Gregoryanz, Nature (London) 529, 63 (2016).

[21] R. P. Dias and I. F. Silvera, Science 355, 715 (2017).

[22] M. I. Eremets and A. P. Drozdov, arXiv:1702.05125.

[23] P. Loubeyre, F. Occelli, and P. Dumas, arXiv:1702.07192.

[24] X.-D. Liu, P. Dalladay-Simpson, R. T. Howie, B. Li, and E. Gregoryanz, arXiv:1704.07601.

[25] A. F. Goncharov and V. V. Struzhkin, arXiv:1702.04246.

[26] I. Silvera and R. Dias, arXiv:1703.03064.

[27] M. I. Eremets, I. A. Troyan, and A. P. Drozdov, arXiv:1601.04479.

[28] C. J. Pickard and R. J. Needs, Nat. Phys. 3, 473 (2007).

[29] A. Perucchi, L. Baldassarre, P. Postorino, and S. Lupi, J. Phys. Condens. Matter 21, 323202 (2009).

[30] S. Azadi, B. Monserrat, W. M. C. Foulkes, and R. J. Needs, Phys. Rev. Lett. 112, 165501 (2014).

[31] J. M. McMahon and D. M. Ceperley, Phys. Rev. Lett. 106, 165302 (2011).

[32] Z. Schlesinger, R. T. Collins, D. L. Kaiser, and F. Holtzberg, Phys. Rev. Lett. 59, 1958 (1987).

[33] L. D. Rotter, Z. Schlesinger, J. P. McCauley, N. Coustel, J. E. Fischer, and A. B. Smith, Nature (London) 355, 532 (1992).

[34] A. P. Drozdov, M. I. Eremets, I. A. Troyan, V. Ksenofontov, and S. I. Shylin, Nature (London) 525, 73 (2015). 
[35] F. Capitani, B. Langerome, J.-B. Brubach, P. Roy, A. Drozdov, M. I. Eremets, E. J. Nicol, J. P. Carbotte, and T. Timusk, Nat. Phys. 13, 859 (2017).

[36] C.-s. Zha, H. Liu, J. S. Tse, and R. J. Hemley, Phys. Rev. Lett. 119, 075302 (2017).

[37] E. Runge and E. K. U. Gross, Phys. Rev. Lett. 52, 997 (1984).

[38] M. Petersilka, U. J. Gossmann, and E. K. U. Gross, Phys. Rev. Lett. 76, 1212 (1996).

[39] B. Rousseau, A. Eiguren, and A. Bergara, Phys. Rev. B 85, 054305 (2012).

[40] A. B. Migdal, Sov. Phys. JETP 7, 996 (1958).

[41] G. M. Eliashberg, Sov. Phys. JETP 11, 696 (1960).

[42] See Supplemental Material at http://link.aps.org/ supplemental/10.1103/PhysRevLett.120.057402 for more details on the theoretical approach and computational methods, pressure dependence of our results, the fitting of data using the Drude model, and calculations on the Cmca-4 molecular structure, which includes Refs. [7,8,21-26,30-33,35-41,43-52].

[43] I. Errea, B. Rousseau, A. Eiguren, and A. Bergara, Phys. Rev. B 86, 085106 (2012).

[44] J. Ibañez-Azpiroz, B. Rousseau, A. Eiguren, and A. Bergara, Phys. Rev. B 89, 085102 (2014).

[45] N. Marzari and D. Vanderbilt, Phys. Rev. B 56, 12847 (1997)
[46] N. Marzari, A. A. Mostofi, J. R. Yates, I. Souza, and D. Vanderbilt, Rev. Mod. Phys. 84, 1419 (2012).

[47] N. E. Bickers, D. J. Scalapino, R. T. Collins, and Z. Schlesinger, Phys. Rev. B 42, 67 (1990).

[48] H. J. Vidberg and J. W. Serene, J. Low Temp. Phys. 29, 179 (1977).

[49] P. Giannozzi et al., J. Phys. Condens. Matter 21, 395502 (2009).

[50] J. P. Perdew and Y. Wang, Phys. Rev. B 45, 13244 (1992).

[51] A. A. Mostofi, J. R. Yates, G. Pizzi, Y.-S. Lee, I. Souza, D. Vanderbilt, and N. Marzari, Comput. Phys. Commun. 185, 2309 (2014).

[52] J. P. Perdew, K. Burke, and M. Ernzerhof, Phys. Rev. Lett. 77, 3865 (1996).

[53] V. M. Silkin, A. Rodriguez-Prieto, A. Bergara, E. V. Chulkov, and P. M. Echenique, Phys. Rev. B 75, 172102 (2007).

[54] A. Rodriguez-Prieto, V. M. Silkin, A. Bergara, and P. M. Echenique, New J. Phys. 10, 053035 (2008).

[55] I. Errea, A. Rodriguez-Prieto, B. Rousseau, V. M. Silkin, and A. Bergara, Phys. Rev. B 81, 205105 (2010).

[56] H.-K. Mao, Y. Ding, Y. Xiao, P. Chow, J. Shu, S. Lebgue, A. Lazicki, and R. Ahuja, Proc. Natl. Acad. Sci. U.S.A. 108, 20434 (2011).

[57] N. V. Smith, Phys. Rev. B 2, 2840 (1970).

[58] I. G. Gurtubay, B. Rousseau, and A. Bergara, Phys. Rev. B 82, 085113 (2010). 